\title{
Video case: Multiple sessile gastric polyposis in ulcerative colitis patient
}

\author{
Tarik Zaher \\ Tropical Medicine Department, Faculty of Medicine, Zagazig University, Egypt \\ tareqzaher@gmail.com
}

43 years old man presented with epigastric pain and bleeding per rectum. Upper gastrointestinal endoscopy (Video1) revealed multiple sessile gastric polyposis. Histopathological examination of one polyp revealed $H$. pylori related inflammation. Colonoscopy (Video2) detected ulcerations and hyperemic inflammations of the rectal and sigmoid mucosa. Histopathological examination of colonic and rectal biopsies revealed ulcerative colitis with mild activity .Finally the question is: Is there any relation between gastric and colonic lesions? 